\title{
Variational Perturbation Theory of the Confined Hydrogen Atom
}

\section{H. E. Montgomery, Jr.}

Chemistry Department, Centre College, 600 West Walnut Street, Danville, KY 40422-1394, USA. Tel. (859) 238-5403, e-mail mntgmry@centre.edu

Received: 28 March 2001 Accepted: 15 June 2001/ Published: 16 June 2001

\begin{abstract}
Variational perturbation theory was used to solve the Schrödinger equation for a hydrogen atom confined at the center of an impenetrable cavity. Ground state and excited state energies and expectation values calculated from the perturbation wavefunction are comparable in accuracy to results from direct numerical solution.
\end{abstract}

Keywords: Perturbation theory, confined systems, one-electron atoms

\section{Introduction}

Confined quantum mechanical systems are a useful model for simulating the effect of external conditions on an enclosed atom. Over sixty years ago, Michels et al [1] studied a hydrogen atom confined at the center of an impenetrable cavity and calculated the effects of pressure on kinetic energy and polarizability. This model has subsequently been applied to a wide range of physical problems. The interested reader is referred to Varshni [2] and references therein.

Application of Rayleigh-Schrödinger perturbation theory to confined systems is complicated by the lack of closed form zero-order wavefunctions. However, when a zero-order wavefunction can be obtained, variational perturbation theory provides a method to carry the calculation to high order. In this work we partition the Hamiltonian using a method developed by Sternheimer [3] and calculate energies and expectation values over a range of confinement radii. By comparison with results from direct numerical calculations and with exact results at selected confinement radii, the variational perturbation wavefunctions are shown to be highly accurate. We restricted our attention to the $1 s, 2 p$ and $3 d$ states which, as the lowest states of a given angular momentum, are readily calculated by variational procedures. 


\section{Computational Procedures}

For a zero-order wavefunction $\psi_{0}$ that satisfies the symmetry and boundary conditions of the system of interest, Sternheimer [3] defined the zero-order potential

$$
U_{0}=\varepsilon_{0}-\frac{\left(T \psi_{0}\right)}{\psi_{0}}
$$

where $T$ is the kinetic energy operator for the system and $\varepsilon_{0}$ is an arbitrary constant chosen to simplify the potential. The zero-order Hamiltonian $H_{0}$ is then given by

$$
H_{0}=T+U_{0} .
$$

For a Hamiltonian $H$, the perturbation potential $H_{1}$ is

$$
H_{1}=H-\lambda H_{0},
$$

where $\lambda$ is an ordering parameter which will be set equal to 1 at the end of the calculation. HylleraasScherr-Knight variational perturbation theory $[4,5]$ can be used to calculate corrections to the energy and wavefunction and to evaluate expectation values.

For zero-order wavefunctions, we use

$$
\begin{gathered}
\psi(1 s)=N_{1 s}\left(r_{0}-r\right) e^{-\alpha r}, \\
\psi(2 p)=N_{2 p} r\left(r_{0}-r\right) e^{-\alpha r} Y_{10}(\theta, \phi), \\
\psi(3 d)=N_{3 d} r^{2}\left(r_{0}-r\right) e^{-\alpha r} Y_{20}(\theta, \phi),
\end{gathered}
$$

where $N$ is a radial normalization factor, $Y_{l m}(\theta, \phi)$ is a normalized spherical harmonic and $\alpha$ is a variational parameter determined by minimizing $\varepsilon_{0}+\varepsilon_{1}$. The cut-off function $\left(r_{0}-r\right)$, introduced by de Groot and ten Seldam [6], ensures that $\psi\left(r_{0}\right)=0$.

The variational perturbation wavefunctions were constructed from trial functions of the form

$$
\chi_{p}=\left(r_{0}-r\right) e^{-\alpha r} Y_{l m}(\theta, \phi) \sum_{n} a_{n} r^{n} .
$$

A typical calculation used fifteen-term trial functions and was carried through ninth-order in the energy. For small values of $r_{0}$ the higher powers of $r$ contribute little to the energy and the number of terms in the trial function was reduced. All calculations were performed using quadruple precision arithmetic ( 30 decimal digits).

\section{Results and Discussion}

Table 1 gives the energy corrections for the 1 s state over a range of confinement radii. Although the first-order correction is large for small $r_{0}$, the magnitude of the energy corrections for second-order and higher steadily decreases and the energy expansion is in exact agreement with the energies calculated by Goldman and Joslin [7] using direct numerical solution. Similar agreement is observed for the $2 p$ and $3 d$ states. 
Table 1. $\varepsilon_{p} \mathrm{~s}$ in a.u. for the $1 s$ state.

\begin{tabular}{clll}
\hline$p$ & $r_{0}=0.1$ a.u. & $r_{0}=1$ a.u. & $r_{0}=10$ a.u. \\
\hline 0 & -7.876050 & -0.97143442 & -0.56482113 \\
1 & 482.777742 & 3.36201803 & 0.06492013 \\
2 & -6.445089 & -0.01739387 & -0.00008937 \\
3 & 0.583226 & 0.00084025 & -0.00000706 \\
4 & -0.050407 & -0.00004101 & -0.00000131 \\
5 & 0.003841 & 0.00000197 & -0.00000035 \\
6 & -0.000231 & -0.00000010 & -0.00000011 \\
7 & 0.000007 & 0.00000001 & -0.00000004 \\
8 & 0.000001 & & -0.00000002 \\
9 & & & -0.00000001 \\
& & & \\
Total & 468.993040 & 2.37399086 & -0.49999927 \\
\hline
\end{tabular}

The radial wavefunction for a free atom has $n-l-1$ nodes, so the $2 s, 3 p$ and $4 d$ wavefunctions, where $n=l+2$, have a single node at

$$
r_{\text {node }}^{(l+2)}=(l+1)(l+2) .
$$

When $n=l+3$, the free atom wavefunction has two nodes, the innermost of which is at

$$
r_{\text {node }}^{(l+3)}=\frac{1}{2}(l+3)(2 l+3-\sqrt{2 l+3})
$$

Similar nodes can be found for $n=l+4$ and $n=l+5$.

If $r_{0}$ for a confined atom corresponds to a node in the radial wavefunction of the free atom with the same value of $l$, the confined atom and the free atom have the same energy. Thus, the $1 s$ energy of the confined atom is -0.125 a.u. at $r_{0}=2$ a.u. and -.05555556 a.u. at $r_{0}=1.90192379$ a.u..

Varshni [8] defined the critical cage radius $r_{c}$ as the radius of the confining sphere at which the total energy of the atom becomes zero. Sommerfeld and Welker [9] showed that $r_{c}$ could be obtained from the zeros of $J_{p}(z)$, the Bessel function of the first kind of order $p$. If $j_{p, i}$ denotes the ith zero of $J_{p}(z)$,

$$
r_{c}(n, l)=\frac{1}{8}\left(j_{2 l+1, n-l}\right)^{2} .
$$

For each state, exact energies can be found for three values of $r_{0}$ either from simple algebra or from tables of Bessel function zeros.

In order to assess the accuracy of the variational perturbation technique, we calculated the variational perturbation energies at $r_{c}, r_{\text {node }}^{(l+2)}$ and $r_{\text {node }}^{(l+3)}$ for the $1 \mathrm{~s}, 2 p$ and $3 d$ states. Rather than tabulate 
nearly identical numbers, we note that with the input radius specified to $1 \times 10^{-10}$ a.u., the exact energy and the ninth-order variational perturbation energy agree to within $1 \times 10^{-10}$ a.u.

Radial operators such as $r$ and $r^{2}$ depend on the wavefunction in regions of configuration space other than those which determine the energy. By comparing expectation values calculated using an approximation method with those calculated by direct solution, we get additional information on the accuracy of the approximate wavefunction. Table 2 gives variational perturbation expectation values for $r^{-1}, r$ and $r^{2}$ for the $1 s, 2 p$ and $3 d$ states over a range of $r_{0}$ from 1 to 8 a.u.

Table 2. Variational perturbation expectation values for the confined hydrogen atom.

\begin{tabular}{|c|c|c|c|c|}
\hline & $r_{0}$ a.u. & $\left\langle r^{-1}\right\rangle$ & $\langle r\rangle$ & $\left\langle r^{2}\right\rangle$ \\
\hline \multirow[t]{8}{*}{$1 s$} & 1 & 2.699146 & 0.468318 & 0.253128 \\
\hline & 2 & 1.535162 & 0.859353 & 0.874825 \\
\hline & 3 & 1.196039 & 1.153206 & 1.624769 \\
\hline & 4 & 1.068129 & 1.341710 & 2.270311 \\
\hline & 5 & 1.020951 & 1.440026 & 2.684972 \\
\hline & 6 & 1.005631 & 1.480911 & 2.887442 \\
\hline & 7 & 1.001347 & 1.494734 & 2.965548 \\
\hline & 8 & 1.000295 & 1.498697 & 2.990632 \\
\hline \multirow[t]{8}{*}{$2 p$} & 1 & 1.894590 & 0.581592 & 0.363464 \\
\hline & 2 & 0.972343 & 1.141079 & 1.405665 \\
\hline & 3 & 0.667442 & 1.675175 & 3.045405 \\
\hline & 4 & 0.517234 & 2.180113 & 5.188390 \\
\hline & 5 & 0.429230 & 2.651681 & 7.726191 \\
\hline & 6 & 0.372629 & 3.085354 & 10.536369 \\
\hline & 7 & 0.334245 & 3.476563 & 13.484712 \\
\hline & 8 & 0.307473 & 3.821145 & 16.430637 \\
\hline \multirow[t]{8}{*}{$3 d$} & 1 & 1.649154 & 0.642711 & 0.432834 \\
\hline & 2 & 0.832952 & 1.275252 & 1.706763 \\
\hline & 3 & 0.561312 & 1.896775 & 3.782271 \\
\hline & 4 & 0.425847 & 2.506340 & 6.615972 \\
\hline & 5 & 0.344883 & 3.102913 & 10.160199 \\
\hline & 6 & 0.291199 & 3.685353 & 14.362564 \\
\hline & 7 & 0.253131 & 4.252409 & 19.165526 \\
\hline & 8 & 0.224849 & 4.802716 & 24.505985 \\
\hline
\end{tabular}


After a literature survey found few high accuracy expectation values, we decided to calculate a representative set of accurate expectation values. Employing the procedures of Ley-Koo and Rubinstein [10] to solve the Schrödinger equation directly, we used the resulting power series expansions to calculate expectation values. To the accuracy given in Table 2, the expectation values from variational perturbation theory are in exact agreement with the results from direct numerical solution. Thus we conclude that the variational perturbation wavefunctions accurately represent the true wavefunction.

Since the $n$ th-order wavefunction gives the energy though order $2 n+1$ and the expectation values only through order $n$, expectation value calculations require higher order wavefunctions than do energy calculations. For example, for the $1 s\left(r_{0}=1\right)$ calculation shown in Table 1 , the $\psi_{p} s$ through $p=3$ contribute all of the perturbation energies greater than $1 \times 10^{-8}$ au. For the expectation values of $1 s\left(r_{0}=1\right)$, it is necessary to calculate the $\psi_{p}$ s through $p=6$ to account for the all of the expectation value coefficients greater than $1 \times 10^{-6}$ au.

From the above examples we conclude that variational perturbation theory provides a simple, efficient procedure for calculating properties of the confined hydrogen atom. The techniques of this paper can be readily extended to other confined polynomial potentials or, by imposing the appropriate orthogonality constraints, to additional excited states.

\section{References}

1. Michels; A.; de Boer; J.; Bijl, A. Remarks concerning molecular interaction and their influence on the polarisability. Physica 1937, 4, 981 - 994.

2. Varshni, Y. P. Accurate wavefunctions for the confined hydrogen atom at high pressures. J. Phys. B: At. Mol. Opt. Phys. 1997, 30, L589 - 1593.

3. Sternheimer, R. M. Electronic polarizabilities of ions from the Hartree-Fock wave fucntions. Phys. Rev. 1954, 96, 951 - 968.

4. Hylleraas, E. A. Über den Grundterm der Zweielectronenprobleme von $\mathrm{H}^{+}, \mathrm{He}, \mathrm{Li}^{+}, \mathrm{Be}^{++}$usw. Z. Physik 1930, 65, 209 - 225. Translated in Hettema, H. Quantum Chemistry, World Scientific: Singapore, 2000, 124 - 139.

5. Scherr, C. W.; Knight, R.E. Two-electron atoms III. A sixth-order perturbation Study of the $1^{1} S$ ground state. Rev. Mod. Phys. 1963, 35, 436 - 442.

6. de Groot, S.R.; ten Seldam, C. A. On the energy levels of a model of the compressed hydrogen atom. Physica 1946, 12, 669 - 682.

7. Goldman, S.; Joslin, C. Spectroscopic properties of an isotopically compressed hydrogen atom. $J$. Phys. Chem. 1992, 96, 6021 - 6027.

8. Varshni, Y. P. Critical cage radii for a confined hydrogen atom. J. Phys. B: At. Mol. Opt. Phys. 1998, 31, 2849 - 2856.

9. Sommerfeld, A.; Welker, H. Künstliche Grenzbedingungen beim Keplerproblem. Ann. Phys. 1938, 32, 56 - 65.

10. Ley-Koo, E.; Rubinstein, S. The hydrogen atom within spherical boxes with penetrable walls. $J$. 
Chem. Phys. 1979 71, 351 - 357.

(C) 2001 by MDPI (http://www.mdpi.org), Basel, Switzerland. 